\title{
Subbalancing Numbers
}

\author{
Ravi Kumar Davala* and G. K. Panda \\ National Institute of Technology, Rourkela, India \\ ${ }^{*}$ Corresponding author: davalaravikumar@gmail.com
}

Article history

Received: 14 June 2016

Received in revised form: 26 April 2018

Accepted: 26 April 2018

Published on line: 1 June 2018

\begin{abstract}
A natural number $n$ is called balancing number (with balancer $r$ ) if it satisfies the Diophantine equation $1+2+\cdots+(n-1)=(n+1)+(n+2)+\cdots+(n+r)$. However, if for some pair of natural numbers $(n, r), 1+2+\cdots+(n-1)<(n+1)+(n+2)+\cdots+(n+r)$ and equality is achieved after adding a natural number $D$ to the left hand side then we call $n$ a $D$-subbalancing number with $D$-subbalancer number $r$. In this paper, such numbers are studied for certain values of $D$.
\end{abstract}

Keywords Balancing and Lucas-balancing numbers, cobalancing numbers, Supercobalancing numbers

Mathematics Subject Classification 11B39, 11B83

\section{Introduction}

Behera and Panda in [1] stated that a natural number $n$ is called a balancing number with balancer $r$ if

$$
1+2+\cdots+(n-1)=(n+1)+(n+2)+\cdots+(n+r) .
$$

Furthermore, they stated that if $n$ is a balancing number then $8 n^{2}+1$ is a perfect square. The $k^{t h}$ balancing number is denoted by $B_{k}$ and $C_{k}=\sqrt{8 B_{k}^{2}+1}$ is called the $k^{\text {th }}$ Lucas-balancing number [2]. The balancing and Lucas-balancing numbers satisfy the recurrence relation $x_{n+1}=$ $x_{n}-x_{n-1}$ with initial terms $B_{0}=0, B_{1}=1$ and $C_{0}=1, C_{1}=3$ respectively. On other hand, $n$ is called a cobalancing number [3] with cobalancer $r$ if

$$
1+2+\cdots+n=(n+1)+(n+2)+\cdots+(n+r) .
$$

The $n^{\text {th }}$ cobalancing number is denoted by $b_{n}$ and cobalancing numbers satisfy the nonhomogeneous recurrence $b_{1}=0, b_{2}=2, b_{n+1}=6 b_{n}-b_{n-1}+2$. The Binet forms are

$$
B_{n}=\frac{\alpha^{2 n}-\beta^{2 n}}{4 \sqrt{2}}, \quad C_{n}=\frac{\alpha^{2 n}+\beta^{2 n}}{2}, \quad b_{n}=\frac{\alpha^{2 n-1}-\beta^{2 n-1}}{4 \sqrt{2}}-\frac{1}{2} .
$$

34:1 (2018) 163-172 | www.matematika.utm.my | eISSN 0127-9602 | 
where $\alpha=1+\sqrt{2}$ and $\beta=1-\sqrt{2}$.

Rout and Panda [4] generalized the concept of balancing numbers and introduced gap balancing numbers. If $k$ is odd, they call a natural number $n$ a $k$-gap balancing number if

$$
1+2+\cdots+\left(n-\frac{k+1}{2}\right)=\left(n+\frac{k+1}{2}\right)+\left(n+\frac{k+3}{2}\right)+\cdots+(n+r)
$$

for some natural number $r$, which is a $k$-gap balancer corresponding to $n$, while for $k$ even, if

$$
1+2+\cdots+\left(n-\frac{k}{2}\right)=\left(n+\frac{k}{2}+1\right)+\left(n+\frac{k}{2}+2\right)+\cdots+(n+r)
$$

for some natural numbers $n$ and $r$ then they call $2 n+1$ a $k$-gap balancing number and $r$ is the corresponding $k$-gap balancer. In [5], Davala and Panda called $n$, a $D$-supercobalancing number if for a fixed positive integer $D, n$ satisfies the Diophantine equation

$$
1+2+\cdots+n=(n+1)+(n+2)+\cdots+(n+r)+D
$$

for some natural number $r$, which they call as $D$-supercobalancer corresponding to $n$. Panda and Panda [6] defined almost balancing numbers as the values of $n$ satisfying the Diophantine equation

$$
1+2+\cdots+(n-1)+1=(n+1)+(n+2)+\cdots+(n+r)
$$

respectively for some $r$, which they called an almost balancer corresponding to $n$. They observed that there are two classes of almost balancing numbers. The almost balancing numbers admits a generalization. The last term 1 of the left hand side of (1) may be replaced by an arbitrary integer $D$ resulting the definition of subbalancing numbers.

\section{Subbalancing Numbers}

Definition 1 For a fixed positive integer D, we call a positive integer $n$, a D-subbalancing number if

$$
1+2+\cdots+(n-1)+D=(n+1)+(n+2)+\cdots+(n+r)
$$

for some natural number $r$, which we call the D-subbalancer corresponding to D-subbalancing number $n$. If $D$ is a negative integer, say $D=-R$, we call $n$ a $R$-superbalancing number and $r$, a R-superbalancer corresponding to $n$.

Since, without D, the left hand side of (2) is less than the right hand side, we prefer the name subbalancing number for $n$. A similar justification applies when $D$ is negative. Observe that when $D=0$, the above definition coincides with that of balancing numbers; hence, we prefer to exclude the case $D=0$ from the above definition. Let $D>0$ and simplifying equation (2), we get

$$
n^{2}+D=\frac{(n+r)(n+r+1)}{2}
$$

Thus, $n$ is a $D$-subbalancing number then $n^{2}+D$ is a triangular number or, equivalently, $8 n^{2}+8 D+1$ is a perfect square. The $D$-subbalancer $r$ corresponding to $n$ is given by

$$
r=\frac{1}{2}\left[-(1+2 n)+\sqrt{8 n^{2}+8 D+1}\right] .
$$


Observe that the value of $n$ will generally depend on the choice of $D$ and the existence of $n$ is not ascertained for each value of $D$, for example, if $D=7$, then $8 n^{2}+8 D+1=8 n^{2}+57$ is not a perfect square for any natural number $n$. Hence, the choice of $D$ plays a crucial role.

It is well-known that for each positive integer $n, 8 b_{n}^{2}+8 b_{n}+1$ is a perfect square. A variant of this result is given in the following lemma.

Lemma 1 For $m \in \mathbb{N}$,

$$
\begin{aligned}
& B_{m}^{2}+b_{2 m}=\frac{1}{2}\left[\frac{B_{m-1}+5 B_{m}-1}{2}\right]\left[\frac{B_{m-1}+5 B_{m}+1}{2}\right] \\
& B_{m}^{2}+b_{2 m+1}=\frac{1}{2}\left[\frac{B_{m+1}+5 B_{m}-1}{2}\right]\left[\frac{B_{m+1}+5 B_{m}+1}{2}\right]
\end{aligned}
$$

Proof Since $8 B_{m}^{2}+1=C_{m}^{2}$ and $B_{m-1}=3 B_{m}-C_{m}$, we have

$$
\begin{aligned}
\left(B_{m-1}+5 B_{m}\right)^{2}-C_{m}^{2} & =\left(B_{m-1}+5 B_{m}\right)^{2}-\left(3 B_{m}-B_{m-1}\right)^{2} \\
& =16\left(B_{m-1} B_{m}+B_{m}^{2}\right) \\
& =16\left[\frac{\alpha^{2 m-2}-\beta^{2 m-2}}{4 \sqrt{2}} \cdot \frac{\alpha^{2 m}-\beta^{2 m}}{4 \sqrt{2}}+\left(\frac{\alpha^{2 m}-\beta^{2 m}}{4 \sqrt{2}}\right)^{2}\right] \\
& =\frac{1}{2}\left[\alpha^{4 m-2}+\beta^{4 m-2}-\alpha^{2}-\beta^{2}+\alpha^{4 m}-2+\beta^{4 m}\right] \\
& =\frac{1}{2}\left[2 \sqrt{2} \alpha^{4 m-1}-2 \sqrt{2} \beta^{4 m-1}-8\right] \\
& =8 b_{2 m},
\end{aligned}
$$

hence (1) follows. The proof of (2) follows from

$$
\begin{aligned}
\left(B_{m+1}+5 B_{m}\right)^{2}-C_{m}^{2} & =\left(B_{m+1}+5 B_{m}\right)^{2}-\left(3 B_{m}-B_{m-1}\right)^{2} \\
& =16\left(B_{m+1} B_{m}+B_{m}^{2}\right) \\
& =16\left[\frac{\alpha^{2 m+2}-\beta^{2 m+2}}{4 \sqrt{2}} \cdot \frac{\alpha^{2 m}-\beta^{2 m}}{4 \sqrt{2}}+\left(\frac{\alpha^{2 m}-\beta^{2 m}}{4 \sqrt{2}}\right)^{2}\right] \\
& =\frac{1}{2}\left[\alpha^{4 m+2}+\beta^{4 m+2}-\alpha^{2}-\beta^{2}+\alpha^{4 m}-2+\beta^{4 m}\right] \\
& =\frac{1}{2}\left[2 \sqrt{2} \alpha^{4 m+1}-2 \sqrt{2} \beta^{4 m+1}-8\right] \\
& =8 b_{2 m+1} .
\end{aligned}
$$

The above lemma ensures the existence of subbalancing numbers when $D$ is restricted to cobalancing numbers.

If $k=1$, then $b_{k}=0$ and the concept of $b_{1}$-subbalancing numbers coincides with that of balancing numbers. The definition of subbalancing numbers excludes this case. If $k=2$ then $b_{k}=2$ and the requirement for a positive integer $n$ to be a $b_{2}$-subbalancing number is that $8 n^{2}+17$ be a perfect square. But according to Rout and Panda [4], such numbers are 3-gap balancing numbers and are of the form $5 B_{n} \pm C_{n}$. 


\section{$2.1 \quad b_{3}$-subbalancing Numbers}

By virtue of Definition 1 , a natural number $n$ is a $b_{3}$-subbalancing number if

$$
1+2+\cdots+(n-1)+14=(n+1)+(n+2)+\cdots+(n+r)
$$

for some natural number $r$, which is a $b_{3}$-subbalancer corresponding to $n$.

Example 1 Since $0+14=2+3+4+5$, we accept 1 as a $b_{3}$-subbalancing number with $b_{3}$-subbalancer 4 . Further $1+2+\cdots+7+14=9+10+11+12,8$ is a $b_{3}$-subbalancing number with $b_{3}$-subbalancer 4 .

It follows from equations (3) and (4) that, if $n$ is a $b_{3}$-subbalancing number then the corresponding $b_{3}$-subbalancer is

$$
r=\frac{-(2 n+1)+\sqrt{8 n^{2}+113}}{2} .
$$

From the above discussion, we notice that if $x$ is a $b_{3}$-subbalancing number then $8 x^{2}+113$ is a perfect square. Since 113 is prime, the congruence

$$
121 x^{2} \equiv 8 x^{2}+113(\bmod 113)
$$

gives

$$
11 x \equiv \pm \sqrt{8 x^{2}+113}(\bmod 113) .
$$

Thus, $\frac{11 x+\sqrt{8 x^{2}+113}}{113}$ or $\frac{11 x-\sqrt{8 x^{2}+113}}{113}$ is a natural number. Since

$$
8\left[\frac{11 x \pm \sqrt{8 x^{2}+113}}{113}\right]^{2}+1=\left[\frac{11 \sqrt{8 x^{2}+113} \pm 8 x}{113}\right]^{2}
$$

it follows that either

$$
\frac{11 x+\sqrt{8 x^{2}+113}}{113} \text { or } \frac{11 x-\sqrt{8 x^{2}+113}}{113}
$$

is a balancing number [1, p.98] Letting

$$
B=\frac{11 x \pm \sqrt{8 x^{2}+113}}{113}
$$

we obtain

$$
(113 B-11 x)^{2}=8 x^{2}+113,
$$

which leads to the quadratic equation

$$
x^{2}-22 B x+113 B^{2}-1=0 .
$$

whose solutions are $x=11 B \pm C,\left(C\right.$ is the Lucas-balancing, $\left.C=\sqrt{8 B^{2}+1}\right)$. We further observe that

$$
8(11 B \pm C)^{2}+113=(8 B \pm 11 C)^{2} .
$$

Thus, the $b_{3}$-subbalancing numbers are of the form $11 B \pm C$ and hence the set

$$
\left\{11 B_{l}+C_{l}, 11 B_{l+1}-C_{l+1}: l=0,1, \cdots\right\}
$$


lists all the $b_{3}$-subbalancing numbers.

The above discussion confirms that the set in (5) is the exhaustive list of $b_{3}$-subbalancing numbers and hence we have the following theorem:

Theorem 1 The $b_{3}-$ subbalancing numbers partition in two classes of the form $11 B_{l}+C_{l}, 11 B_{l+1}-$ $C_{l+1}, l \geq 0$.

\subsection{Computation of $b_{5}$-subbalancing Numbers}

In view of the definition 1 , a natural number $n$ is a $b_{5}$-subbalancing number if

$$
1+2+\cdots+(n-1)+492=(n+1)+(n+2)+\cdots+(n+r)
$$

for some natural number $r$, which is the $b_{5}$-subbalancer corresponding to $n$.

Example 2 From the following examples 2,6,47 and 57 are $b_{5}$-subbalancing numbers with $29,26,26$ and 29 as corresponding $b_{5}$-subbalancers.

1. $1+492=3+4+\cdots+31$

2. $1+2+3+4+5+492=7+8+\cdots+32$

3. $1+2+\cdots+46+492=48+49+\cdots+73$

4. $1+2+\cdots+56+492=58+59+\cdots+86$

It is easy to see that if $n$ is a $b_{5}$-subbalancing number then the corresponding $b_{5}$-subbalancer is $r=\frac{1}{2}\left[-(2 n+1)+\sqrt{8 n^{2}+3937}\right]$. We infer from the above discussion that, if $x$ is a $b_{5^{-}}$ subbalancing number then $8 x^{2}+3937$ is a perfect square. Thus, computation of $b_{5}$-subbalancing numbers reduces to solving the Diophantine equation

$$
8 x^{2}+3937=y^{2} .
$$

To find all the $b_{5}$-subbalancing numbers one needs to solve the generalized Pell's equation $y^{2}-8 x^{2}=3937$. The bounds for its fundamental solutions are $|y| \leq \sqrt{7874}<89$ and $0 \leq x \leq$ $\sqrt{3937 / 8}<23$ ( see [7]). Thus, we need to find those integers $x$ in the interval $[0,23)$ such that $8 x^{2}+3937$ is a perfect square. This happens for $(x, y)=(2, \pm 63)$ and $(6, \pm 65)$ from which it is easy to see that there are four fundamental solutions $-63+2 \sqrt{8},-65+6 \sqrt{8}, 63+2 \sqrt{8}$ and $65+6 \sqrt{8}$ respectively. Using techniques similar to that used in [6], we get the following four classes of solutions for $b_{5}$-subbalancing numbers in terms of balancing and Lucas-balancing numbers:

$$
\left\{63 B_{l}+2 C_{l}, 63 B_{l+1}-2 C_{l+1}, 65 B_{l}+6 C_{l}, 65 B_{l+1}-6 C_{l+1}: l=0,1, \cdots\right\}
$$

Thus, we have the following theorem:

Theorem 2 The $b_{5}$-subbalancing numbers can be classified in four classes of the form $63 B_{l}+$ $2 C_{l}, 63 B_{l+1}-2 C_{l+1}, 65 B_{l}+6 C_{l}, 65 B_{l+1}-6 C_{l+1}: l \geq 0$. 
From the above discussion, it is clear that for some values of $k$, there can be more than two classes of $b_{k}$-subbalancing numbers. Obtaining each class is a difficult task; however, we employ the same techniques used by the author in [5] for obtaining two classes of solutions.

In the following theorems we explore two classes of $b_{k}$-subbalancing numbers corresponding to even and odd positive integer $k$.

Theorem 3 For $m>1$, the values of $x$ satisfying the Diophantine equation

$$
1+2+\cdots+(x-1)+b_{2 m}=(x+1)+(x+2)+\cdots+(x+r)
$$

for some $r$, may result in multiple classes. Two such classes of solutions are

$$
\left(B_{m-1}+5 B_{m}\right) B_{l}+B_{m} C_{l} \text { and }\left(B_{m-1}+5 B_{m}\right) B_{l+1}-B_{m} C_{l+1}
$$

for $l \geqslant 0$.

Proof In view of (3), $8 x^{2}+8 b_{2 m}+1$ is perfect square. Since, $8 B_{m}^{2}+8 b_{2 m}+1$ is a perfect square (Lemma 1), the congruence

$$
\left(8 B_{m}^{2}+8 b_{2 m}+1\right) x^{2} \equiv B_{m}^{2}\left(8 x^{2}+8 b_{2 m}+1\right)\left(\bmod 8 b_{2 m}+1\right)
$$

holds. Hence any $x$ satisfying the congruences

$$
\sqrt{8 B_{m}^{2}+8 b_{2 m}+1} x \equiv \pm B_{m} \sqrt{8 x^{2}+8 b_{2 m}+1}\left(\bmod 8 b_{2 m}+1\right)
$$

is also a solution of the congruence (8). Thus, to obtain two classes of $b_{2 m}$-subbalancing numbers, we need to solve the congruences (9).

Equation (9) implies that

$$
\frac{x \sqrt{8 B_{m}^{2}+8 b_{2 m}+1}+B_{m} \sqrt{8 x^{2}+8 b_{2 m}+1}}{8 b_{2 m}+1}
$$

or

$$
\frac{x \sqrt{8 B_{m}^{2}+8 b_{2 m}+1}-B_{m} \sqrt{8 x^{2}+8 b_{2 m}+1}}{8 b_{2 m}+1}
$$

is a natural number. Since

$$
\begin{aligned}
& 8\left[\frac{x \sqrt{8 B_{m}^{2}+8 b_{2 m}+1} \pm B_{m} \sqrt{8 x^{2}+8 b_{2 m}+1}}{8 b_{2 m}+1}\right]^{2}+1 \\
& =\left[\frac{8 x B_{m} \pm \sqrt{8 x^{2}+8 b_{2 m}+1} \sqrt{8 B_{m}^{2}+8 b_{2 m}+1}}{8 b_{2 m}+1}\right]^{2}
\end{aligned}
$$

it follows that either

$$
\frac{x \sqrt{8 B_{m}^{2}+8 b_{2 m}+1}+B_{m} \sqrt{8 x^{2}+8 b_{2 m}+1}}{8 b_{2 m}+1}
$$


or

$$
\frac{x \sqrt{8 B_{m}^{2}+8 b_{2 m}+1}-B_{m} \sqrt{8 x^{2}+8 b_{2 m}+1}}{8 b_{2 m}+1}
$$

is a balancing number [1]. Letting

$$
B=\frac{\left(B_{m-1}+5 B_{m}\right) x \pm B_{m} \sqrt{8 x^{2}+8 b_{2 m}+1}}{8 b_{2 m}+1}
$$

we get

$$
\left[\left(B_{m-1}+5 B_{m}\right) x-B\left(8 b_{2 m}+1\right)\right]^{2}=B_{m}^{2}\left(8 x^{2}+8 b_{2 m}+1\right)
$$

which can be transformed to the quadratic equation

$$
x^{2}-2\left(B_{m-1}+5 B_{m}\right) B x+B^{2}\left(8 b_{2 m}+1\right)-B_{m}^{2}=0
$$

whose solutions are $x=\left(B_{m-1}+5 B_{m}\right) B \pm B_{m} C$. We further observe that

$$
8\left[\left(B_{m-1}+5 B_{m}\right) B \pm B_{m} C\right]^{2}+8 b_{2 m}+1=\left[\left(B_{m-1}+5 B_{m}\right) C \pm 8 B_{m} B\right]^{2} .
$$

Thus, two classes of $b_{2 m}$-subbalancing numbers are $\left(B_{m-1}+5 B_{m}\right) B_{l}+B_{m} C_{l}$ and $\left(B_{m-1}+\right.$ $\left.5 B_{m}\right) B_{l+1}-B_{m} C_{l+1}$ for $l \geq 0$.

Theorem 4 For $m \geq 1$, the values of $x$ satisfying the Diophantine equation

$$
1+2+\cdots+(x-1)+b_{2 m+1}=(x+1)+(x+2)+\cdots+(x+r)
$$

may result in multiple classes. Two such classes of solutions are given by

$$
\left(B_{m+1}+5 B_{m}\right) B_{l}+B_{m} C_{l} \text { and }\left(B_{m+1}+5 B_{m}\right) B_{l+1}-B_{m} C_{l+1}
$$

for $l \geqslant 0$.

Proof By virtue of equation (3), $8 x^{2}+8 b_{2 m+1}+1$ is perfect square. Since, $8 B_{m}^{2}+8 b_{2 m+1}+1$ is a perfect square (Lemma 1 ), the congruence

$$
\left(8 B_{m}^{2}+8 b_{2 m+1}+1\right) x^{2} \equiv B_{m}^{2}\left(8 x^{2}+8 b_{2 m+1}+1\right)\left(\bmod 8 b_{2 m+1}+1\right)
$$

holds and is implied by

$$
x \sqrt{8 B_{m}^{2}+8 b_{2 m+1}+1} \equiv \pm B_{m} \sqrt{8 x^{2}+8 b_{2 m+1}+1}\left(\bmod 8 b_{2 m+1}+1\right)
$$

and any solution of the latter congruence is a solution of the former and is a $b_{2 m+1}$-subbalancing number. In view of the latter congruence

$$
\frac{x \sqrt{8 B_{m}^{2}+8 b_{2 m+1}+1}+B_{m} \sqrt{8 x^{2}+8 b_{2 m+1}+1}}{8 b_{2 m+1}+1}
$$

or

$$
\frac{x \sqrt{8 B_{m}^{2}+8 b_{2 m+1}+1}-B_{m} \sqrt{8 x^{2}+8 b_{2 m+1}+1}}{8 b_{2 m+1}+1}
$$


is a natural number. Since

$$
\begin{aligned}
& 8\left[\frac{x \sqrt{8 B_{m}^{2}+8 b_{2 m+1}+1} \pm B_{m} \sqrt{8 x^{2}+8 b_{2 m+1}+1}}{8 b_{2 m+1}+1}\right]^{2}+1 \\
& =\left[\frac{8 x B_{m} \pm \sqrt{8 x^{2}+8 b_{2 m+1}+1} \sqrt{8 B_{m}^{2}+8 b_{2 m+1}+1}}{8 b_{2 m+1}+1}\right]^{2}
\end{aligned}
$$

it follows that either

$$
\frac{x \sqrt{8 B_{m}^{2}+8 b_{2 m+1}+1}+B_{m} \sqrt{8 x^{2}+8 b_{2 m+1}+1}}{8 b_{2 m+1}+1}
$$

or

$$
\frac{x \sqrt{8 B_{m}^{2}+8 b_{2 m+1}+1}-B_{m} \sqrt{8 x^{2}+8 b_{2 m+1}+1}}{8 b_{2 m+1}+1}
$$

is a balancing number [1]. Letting

$$
B=\frac{\left(B_{m+1}+5 B_{m}\right) x \pm B_{m} \sqrt{8 x^{2}+8 b_{2 m+1}+1}}{8 b_{2 m+1}+1}
$$

we get

$$
\left[\left(B_{m+1}+5 B_{m}\right) x-B\left(8 b_{2 m+1}+1\right)\right]^{2}=B_{m}^{2}\left(8 x^{2}+8 b_{2 m+1}+1\right),
$$

which, on rearrangement, results in the quadratic equation

$$
x^{2}-2\left(B_{m+1}+5 B_{m}\right) B x+B^{2}\left(8 b_{2 m+1}+1\right)-B_{m}^{2}=0,
$$

whose solutions are $x=\left(B_{m+1}+5 B_{m}\right) B \pm B_{m} C$. We further observe that

$$
8\left[\left(B_{m+1}+5 B_{m}\right) B \pm B_{m} C\right]^{2}+8 b_{2 m+1}+1=\left[\left(B_{m+1}+5 B_{m}\right) C \pm 8 B_{m} B\right]^{2} .
$$

Thus, two classes of $b_{2 m+1}$-subbalancing numbers are $\left(B_{m+1}+5 B_{m}\right) B_{l}+B_{m} C_{l}$ and $\left(B_{m+1}+\right.$ $\left.5 B_{m}\right) B_{l+1}-B_{m} C_{l+1}$ for $l \geq 0$.

In view of the above theorems, for a fixed positive integer $m$, the $b_{2 m}$-subbalancing numbers are given by $x_{2 l}=\left(B_{m-1}+5 B_{m}\right) B_{l}+B_{m} C_{l}, x_{2 l+1}=\left(B_{m-1}+5 B_{m}\right) B_{l+1}-B_{m} C_{l+1}$ and the $b_{2 m+1}$-subbalancing numbers are $x_{2 l}=\left(B_{m+1}+5 B_{m}\right) B_{l}+B_{m} C_{l}, x_{2 l+1}=\left(B_{m+1}+5 B_{m}\right) B_{l+1}-$ $B_{m} C_{l+1}$ for $l \geq 0$. Since balancing and Lucas-balancing numbers satisfy the recurrence relation $y_{n+1}=6 y_{n}-y_{n-1}$, it follows that $b_{m}$-subbalancing numbers satisfy the recurrence relation $x_{n+2}=6 x_{n}-x_{n-2}, n \geq 3$.

In the following theorem we give functions that transforms from balancing to subbalancing numbers and subbalancing numbers to balancing numbers.

Theorem 5 For a balancing number $x, f(x)=\left(B_{m-1}+5 B_{m}\right) x+B_{m} \sqrt{8 x^{2}+1}$ and $g(x)=$ $\left(B_{m-1}+5 B_{m}\right) x-B_{m} \sqrt{8 x^{2}+1}$ are $b_{2 m}$-subbalancing numbers. 
We next find functions that transform $b_{2 m}$-subbalancing numbers to balancing numbers. It is easy to check that the functions are strictly increasing in the domain $[0, \infty)$. Hence their inverses exist and are equal to

$$
f^{-1}(y)=\frac{\left(B_{m-1}+5 B_{m}\right) y-B_{m} \sqrt{8 x^{2}+8 b_{2 m}+1}}{8 b_{2 m}+1}
$$

and

$$
g^{-1}(y)=\frac{\left(B_{m-1}+5 B_{m}\right) y+B_{m} \sqrt{8 x^{2}+8 b_{2 m}+1}}{8 b_{2 m}+1} .
$$

The above discussion leads to the following theorems.

Theorem 6 If $y=\left(B_{m-1}+5 B_{m}\right) B_{n}+B_{m} C_{n}$ is $b_{2 m}$-subbalancing number then

$$
B_{n}=\frac{\left(B_{m-1}+5 B_{m}\right) y-B_{m} \sqrt{8 y^{2}+8 b_{2 m}+1}}{8 b_{2 m}+1}
$$

and

$$
C_{n}=\frac{\left(B_{m-1}+5 B_{m}\right) \sqrt{8 y^{2}+8 b_{2 m}+1}-8 y B_{m}}{8 b_{2 m}+1} .
$$

Theorem 7 If $y=\left(B_{m-1}+5 B_{m}\right) B_{n}-B_{m} C_{n}$ is $b_{2 m}$-subbalancing number then

$$
B_{n}=\frac{\left(B_{m-1}+5 B_{m}\right) y+B_{m} \sqrt{8 y^{2}+8 b_{2 m}+1}}{8 b_{2 m}+1}
$$

and

$$
C_{n}=\frac{\left(B_{m-1}+5 B_{m}\right) \sqrt{8 y^{2}+8 b_{2 m}+1}+8 y B_{m}}{8 b_{2 m}+1} .
$$

Theorem 8 If $x$ is a balancing number then $\alpha(x)=\left(B_{m+1}+5 B_{m}\right) x+B_{m} \sqrt{8 x^{2}+1}$ and $\beta(x)=\left(B_{m+1}+5 B_{m}\right) x-B_{m} \sqrt{8 x^{2}+1}$ are $b_{2 m+1}$-subbalancing numbers.

We next find functions that transform $b_{2 m+1}$-subbalancing numbers to balancing numbers. It is easy to check that the functions are strictly increasing in the domain $[0, \infty)$. Hence their inverses exist and are equal to

$$
\alpha^{-1}(y)=\frac{\left(B_{m+1}+5 B_{m}\right) y-B_{m} \sqrt{8 x^{2}+8 b_{2 m}+1}}{8 b_{2 m+1}+1}
$$

and

$$
\beta^{-1}(y)=\frac{\left(B_{m+1}+5 B_{m}\right) y+B_{m} \sqrt{8 x^{2}+8 b_{2 m}+1}}{8 b_{2 m+1}+1} .
$$

The above discussion leads to the following theorems.

Theorem 9 If $y=\left(B_{m+1}+5 B_{m}\right) B_{n}+B_{m} C_{n}$ is $b_{2 m+1}$-subbalancing number then

$$
B_{n}=\frac{\left(B_{m+1}+5 B_{m}\right) y-B_{m} \sqrt{8 y^{2}+8 b_{2 m}+1}}{8 b_{2 m+1}+1}
$$

and

$$
C_{n}=\frac{\left(B_{m+1}+5 B_{m}\right) \sqrt{8 y^{2}+8 b_{2 m}+1}-8 y B_{m}}{8 b_{2 m+1}+1} .
$$


Theorem 10 If $y=\left(B_{m+1}+5 B_{m}\right) B_{n}-B_{m} C_{n}$ is $b_{2 m+1}$-subbalancing number then

$$
B_{n}=\frac{\left(B_{m+1}+5 B_{m}\right) y+B_{m} \sqrt{8 y^{2}+8 b_{2 m}+1}}{8 b_{2 m+1}+1}
$$

and

$$
C_{n}=\frac{\left(B_{m+1}+5 B_{m}\right) \sqrt{8 y^{2}+8 b_{2 m}+1}+8 y B_{m}}{8 b_{2 m+1}+1} .
$$

\section{Conclusion}

In this paper, we define $D$-subbalancing numbers by restricting $D$ to cobalancing numbers. However, for many values of $D, D$-subbalancer numbers exist. For example, one can verify that a natural number $x$ is a 6 -subbalancing number if and only if $8 x^{2}+49$ is a perfect square and the values of $x$ satisfying $8 x^{2}+49=y^{2}$ are 5-gap balancing numbers [4]. Indeed, finding all feasible values of $D$ is an interesting problem.

\section{References}

[1] Behera, A and Panda, G. K. On the square roots of triangular numbers. Fib. Quart. 1999. 37(2): $98-105$.

[2] Panda, G. K. Some fascinating properties of balancing numbers. In Proc. of Eleventh Internet. Conference on Fibonacci Numbers and Their Applications, Cong. Numerantium. 2009. 194: 185-189.

[3] Panda, G. K. and Ray, P. K. Cobalancing numbers and cobalancers. Int. J. Math. Math. Sci. 2005. 8: 1189-1200.

[4] Rout, S. S. and Panda, G. K. k-gap balancing numbers. Period. Math. Hungar. 2015. 70(1): $109-121$.

[5] Davala, R. K. and Panda, G. K. Supercobalancing numbers. MATEMATIKA. 2016. 32(1): $31-42$.

[6] Panda, G. K. and Panda, A. K. Almost balancing numbers. Indian Math. Soc. 2015. 82(34): $147-156$.

[7] Mollin, R. A. Fundamental Number Theory with Applications. London: CRC Press. 2004. 